\title{
Transition States for Shock Induced Chemical Reactions in Binary Energetic Materials
}

\author{
Vindhya Narayanan*, Sathya Hanagud \\ Georgia Institute of Technology, Atlanta, GA, USA \\ Email: ^hanagud@ae.gatech.edu
}

How to cite this paper: Narayanan, V. and Hanagud, S. (2020) Transition States for Shock Induced Chemical Reactions in Binary Energetic Materials. Journal of Materials Science and Chemical Engineering, 8, 27-35.

https://doi.org/10.4236/msce.2020.87003

Received: June 19, 2020

Accepted: July 27, 2020

Published: July 30, 2020

Copyright $\odot 2020$ by author(s) and Scientific Research Publishing Inc. This work is licensed under the Creative Commons Attribution International License (CC BY 4.0).

http://creativecommons.org/licenses/by/4.0/

\begin{abstract}
In this paper, numerical simulation of shock induced chemical reactions of a thermite mixture of binary energetic material, aluminum and iron oxide, are discussed. To initiate the chemical reactions, the impact or the shockwave should supply the energy to take the reactants, aluminum and iron oxide, to the transition state. Thus, for any numerical simulation of the shock or impact induced chemical reaction in a continuum or mesoscale, it is necessary to identify the transition state. The transition state for the thermite mixture, of aluminum and iron oxide, is identified in this paper and a result from a numerical simulation of the shock induced chemical reaction, in a continuum scale is presented.
\end{abstract}

\section{Keywords}

Numerical Simulation, Chemical Reaction

\section{Introduction and Significance of the Need for Transition States}

Transition states are needed if we are studying catalytic reactions with microkinetic theory [1] [2] or we are exploring shock induced chemical reactions in binary energetic materials that are also known as reactive materials. Shock waves create a unique environment of high pressure, high temperature, large strains and high strain-rates. It has been observed that shock induced chemical reactions, of certain mixtures of metal and metal oxide or intermetallic mixtures, are exothermic and can lead to the synthesis of new materials [3] [4] [5] [6] that are not possible under other conditions. This observation of exothermic reactions resulted in the development of materials that could be used as binary energetic materials or reactive materials [7] [8] [9] [10] [11]. The binary energetic materials are of significant interest to the energetic materials commu- 
nity because of their capability of releasing high heat content during chemical reactions and the relative insensitivity of these types of energetic materials. These binary mixtures of energetic materials also provide an opportunity to develop a dual functional material with both strength and energetic characteristics.

To design systems with these dual functional materials, it is necessary to integrate the steps of the synthesis, determination of properties and evaluation of shock induced or thermal chemical reaction, as needed in the application. The success of the design of the system, to a significant extent, depends on the last step of the chemical reaction. To successfully study the shock induced or thermally induced chemical reaction, numerically, we need transition states. However, to date, transition states in the chemical reaction of metal-metal oxide mixtures such as aluminum and iron oxide, are not understood. Thus, the significance of this work is to discuss techniques to obtain the transition states that have applications in the field of design of systems with dual functional materials such as metal-metal oxides or other reactive materials.

In binary mixtures, such as aluminum and iron oxide, aluminum is the fuel and iron-oxide $\left(\mathrm{Fe}_{2} \mathrm{O}_{3}\right)$ is the oxidizer. Such a mixture of aluminum and iron-oxide can release a significant amount of energy through an exothermic reaction when the mixture is subjected to a shock or thermal loading. In addition to binary energetic materials, such as $\mathrm{Al}-\mathrm{Fe}_{2} \mathrm{O}_{3}$ mixture, there are other metal/metal mixtures that can be used as the binary energetic material [12]. The word "thermite" was first used to describe the reduction of a metal-oxide through an exothermic reaction [13].

\section{Reaction Mechanisms}

Specific shock induced reaction mechanism for mixtures of metal and metal oxide has not been established and needs ab initio studies with supercomputers [14]. However, many different explanations have been proposed for the initiation of chemical reaction in binary metal/metal-oxide energetic materials and metal and metal mixtures, also known as intermetallic mixtures. Two such proposed mechanisms are the thermo-chemical model and the mechano-chemical model. In the thermochemical model, the thermodynamic characteristics of the system are assumed to result in an elevated temperature that will drive the chemical reaction with associated high stresses. Boslough [15] experimentally measured the shock temperature in a thermite mixture with $50 \%$ porosity using radiation pyrometry. Based on the results, he observed an initial spike in the temperature when the mixture is shock-impacted. This spike is attributed to the collapse of pores in the mixture. This increased temperature then leads to a chemical reaction initiation. Furthermore, Boslough attributed that the dynamic mixing, which occurs at the shock front, controls the rate of the chemical reaction. Thermochemical models suggest that reactions take place in a very thin region of shock wave while, it has been found that the reactions occur over a wide region and covers a time duration of up to $100 \mathrm{~ns}$ [16]. A thermodynamic equilibrium is assumed in this model. 
The second model that explains the initiation of chemical reactions is the mechano-chemical model. According to this model, inter-particle contacts result in high stresses and enhanced plastic deformations leading to a" more intimately" mixed condition, and the resulting reactions. Plastic deformation is assumed to enhance the solid-state reactivity. Thadhani et al. [17] experimentally studied the particle size effects on shock-induced chemical reactions. From their observations, they attributed the initiation of chemical reactions in shock compression of powder mixtures of $\mathrm{Ti}$ and $\mathrm{Si}$ to the solid-state mechanochemical processes and explained the initiation of chemical reaction, based on plastic flow and mechanical deformations around the voids. They also studied [18] several mixtures such as $\mathrm{Ti}-\mathrm{Si}$, $\mathrm{Ti}-\mathrm{Al}$ and $\mathrm{Ti}-\mathrm{B}$ and subsequently showed that the chemical reaction initiation and the reaction rate significantly vary with the mechanical properties of the reactants.

Other discussion, from materials science community, concerns whether shock wave induces the chemical reaction or indirectly assists the reaction. Thus, the two different reaction mechanisms are defined as the shock-induced or shock-initiated chemical reactions; and the shock-assisted chemical reactions. Shock-assisted chemical reactions are thermally initiated reactions that occur through an increase in the bulk temperature due to the passage of a shock wave. The reactions are also assumed to occur in time-scales of "thermal equilibrium" while shock-induced chemical reactions are initiated by mechanisms resulting from the increase of pressure or stress and are assumed to occur in time scales associated with "pressure equilibrium" [19]. In other words, the shock-assisted chemical reactions are assumed to occur via the thermochemical model and the shock-induced chemical reactions via the mechanochemical model. And solidification process and the pressure-initiated microstructure being defined by the deformation, plastic flow and enhanced mixing between the components. Meyers et al. [20] experimentally studied shock-assisted and shock-induced reactions for Nb-Si and Mo-Si mixtures. They modified the Krueger-Vreeland threshold energy by including a term that denoted the plastic deformation and showed that plastic deformation or intense shear localization plays an important role and can trigger chemical reactions. Their results seemed to indicate that the reactions in this case were more shock-induced rather than shock-assisted.

\section{Transition State:}

As discussed in the last section, there are both experimental studies and theoretical studies to understand the reactions mechanisms that explain the shock induced chemical reactions in mixtures of metal and metal oxides or metal-metal mixtures. The theoretical studies include supercomputer simulations at atomistic level. However, most practical applications need a numerical simulation of shock or impact induced simulations of chemical reactions in a continuum or meso scale. For mixtures of metal and metal oxides such as aluminum and iron oxide, one option is to use a uniformly blended mixture theory. Then, in a continuum, every point can contain reactants (aluminum and iron oxide); voids; reaction products (iron and aluminum oxide); and transition states. Then, 
we formulate conservation equation for mixture variables (mass, momentum and energy equations), mixture constitutive equations and modified reaction equations (modified Arrhenius equations), in the framework of equilibrium and non-equilibrium thermodynamics. We know the reactants and products, but we do not know the transition state. Thus, prior to any numerical simulation of shock induced chemical reactions, it is necessary to determine the transition states.

Mixtures like $\mathrm{Al}$ and $\mathrm{Fe}_{2} \mathrm{O}_{3}$ do not react at the room temperature. The composite can react if it is supplied with energy to take the system of reactants to the transition state. This energy should be supplied.

In the case of shock-induced chemical reactions, the energy is supplied by the shock wave acting on the composite. In practice, two different deformation mechanisms can supply the energy as a result of the shock propagation in the composite. One of the mechanisms is the irreversible pore collapse that can result in heat and energy supply to the composite from the shock loading. The second mechanism is through the plastic work resulting from the shock propagation through the composite. In either case or a combination of the two cases, it is necessary that additional energy $E_{\alpha}$ is supplied to the composite before a reaction can start. From Figure 1, one can note that

$$
E_{\alpha}>E_{\text {reactants }}>E_{\text {products }}
$$

According to the theory of transition states, there can be multiple states that the reaction can encounter as it proceeds from the reactants to the products. The one-dimensional projection of this path is depicted in Figure 2. This figure also shows the projection of the multiple transition states to a single transition state, which is usually used. At the continuum level, the transition state can be identified from energy considerations. There are two points that need to be remembered. Firstly, the energy of the transition state is higher than the energy of both the reactants as well as the products. Given multiple options for the transition state, the reactants will first reach the state that has the lowest energy among the various possibilities. Thus, the probability that the transition state is the one, among all the options, with the lowest energy, but is higher than the energy of reactants and products. This state is then chosen as the transition state for the reaction. The specific calculations identifying the transition state for the thermite mixture of aluminum and iron-oxide is now presented.

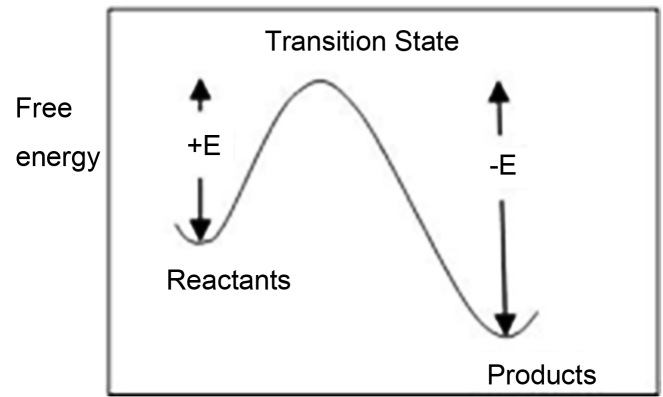

Figure 1. The reaction path from reactants to products. 


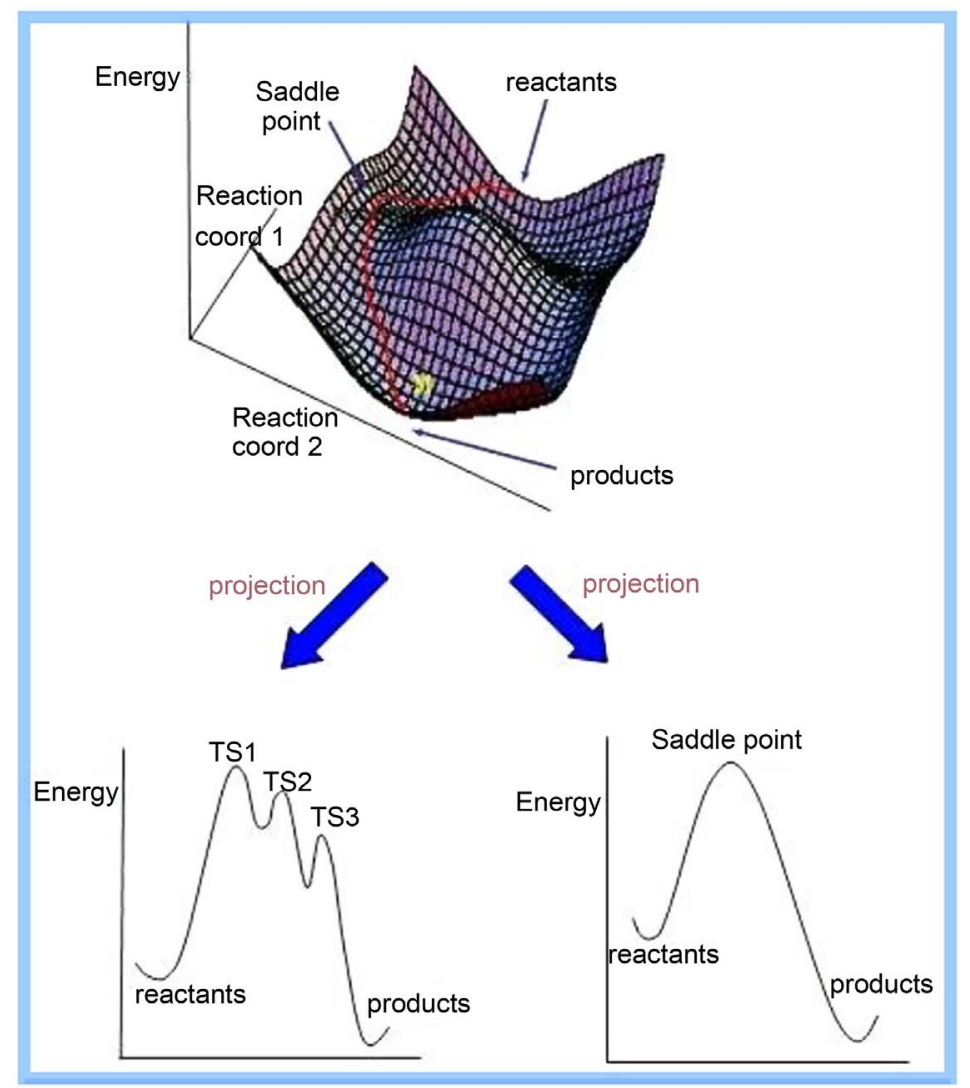

Figure 2. Multiple transition states.

\section{Identification of Transition States for the Reaction of Aluminum and Iron Oxide}

In order to study the shock-related chemical reactions in an energetic mixture, it is necessary to know the activation energy required for the reaction. The activation energy is the difference in energy between the reactants and the transition state. In order to obtain the activation energy, it is necessary to first identify the transition state. It is identified based on energy considerations. The reaction between aluminum and iron-oxide gives iron and aluminum-oxide is as follows:

$$
2 \mathrm{Al}+\mathrm{Fe}_{2} \mathrm{O}_{3} \rightarrow \mathrm{Al}_{2} \mathrm{O}_{3}+2 \mathrm{Fe}
$$

The reaction is assumed to go to completion with no additional products formed other than iron and aluminum-oxide. Different combinations of $\mathrm{Al}, \mathrm{O}$ and $\mathrm{Fe}$ that can exist in the solid state, in nature, and that which can be formed by a stoichiometric reaction between $\mathrm{Al}$ and $\mathrm{Fe}_{2} \mathrm{O}_{3}$ were considered by looking at the database of chemical analysis. The possible transition species are: (AlO, $\left.\mathrm{Al}_{2} \mathrm{O}\right)$ and $(\mathrm{FeO}, \mathrm{Fe})$. Considering combinations of these transition species, four reaction paths are possible. They are:
1) $2 \mathrm{Al}+\mathrm{Fe}_{2} \mathrm{O}_{3} \rightarrow \mathrm{AlO}+2 \mathrm{FeO}+\mathrm{Al} \rightarrow \mathrm{Al}_{2} \mathrm{O}_{3}+2 \mathrm{Fe}$
2) $2 \mathrm{Al}+\mathrm{Fe}_{2} \mathrm{O}_{3} \rightarrow 2 \mathrm{AlO}+1.3 \mathrm{Fe}+0.3 \mathrm{Fe}_{2} \mathrm{O}_{3} \rightarrow \mathrm{Al}_{2} \mathrm{O}_{3}+2 \mathrm{Fe}$
3) $2 \mathrm{Al}+\mathrm{Fe}_{2} \mathrm{O}_{3} \rightarrow \mathrm{Al}_{2} \mathrm{O}+0.6 \mathrm{Fe}+0.7 \mathrm{Fe}_{2} \mathrm{O}_{3} \rightarrow \mathrm{Al}_{2} \mathrm{O}_{3}+2 \mathrm{Fe}$
4) $2 \mathrm{Al}+\mathrm{Fe}_{2} \mathrm{O}_{3} \rightarrow \mathrm{Al}_{2} \mathrm{O}+2 \mathrm{FeO} \rightarrow \mathrm{Al}_{2} \mathrm{O}_{3}+2 \mathrm{Fe}$ 
The transition state has a higher energy than both the reactants and the products. It is the saddle point in the reaction path and has the highest energy. At the same time, the products that are formed are a more energetically favorable state than the reactants and thus have a lower energy state than the reactants. The Gibbs free energy for the reactants, products and the four transition states were plotted (Figure 3). Two of the transition states have energies that are between the energies of the reactants and those of the products. Hence, these two states cannot be the transition states of the thermite reaction between aluminum and iron-oxide. The other two transition states $\mathrm{AlO}+2 \mathrm{FeO}+\mathrm{Al}$ and $\mathrm{Al}_{2} \mathrm{O}+0.6 \mathrm{Fe}+$ $0.7 \mathrm{Fe}_{2} \mathrm{O}_{3}$ were found to satisfy the energy considerations. In addition, it was found that $\mathrm{Al}_{2} \mathrm{O}+0.6 \mathrm{Fe}+0.7 \mathrm{Fe}_{2} \mathrm{O}_{3}$ has a higher energy state than $\mathrm{AlO}+2 \mathrm{FeO}+$ Al. Thus, in a chemical reaction, a greater input of energy would be required to reach the former transition state as opposed to the latter. Hence, the probability of the reaction proceeding through $\mathrm{AlO}+2 \mathrm{FeO}+\mathrm{Al}$ as the energy that needs to be supplied to attain this state is lower of the two possibilities. We are considering a single transition state and have a one-step reaction mechanism. The reaction mechanism was identified to be

$$
2 \mathrm{Al}+\mathrm{Fe}_{2} \mathrm{O}_{3} \rightarrow \mathrm{AlO}+2 \mathrm{FeO}+\mathrm{Al} \rightarrow \mathrm{Al}_{2} \mathrm{O}_{3}+2 \mathrm{Fe}
$$

This transition state agrees with experiments by Peiris and Gump [21]. They studied the laser-initiated reactions in several thermite mixtures. In their analysis of the thermite mixture, they found evidence of the compound AlO. Figure 4 shows the results they obtained at various intervals of time. It also shows the time intervals when $\mathrm{AlO}$ was observed.

Now that the transition state has been identified, the next step is to calculate the energy difference between the transition state and the reactants. This would give us the activation energy necessary for the reaction. The activation energy for the reaction is calculated from the Gibbs free energy to reach the transition state

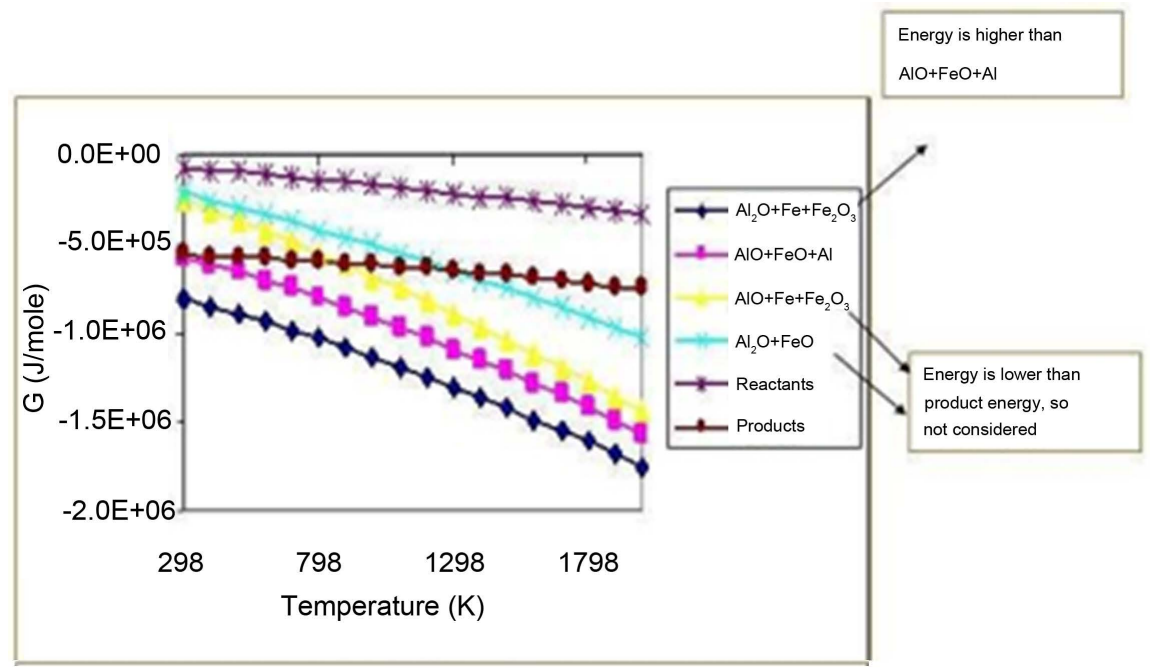

Figure 3. Plot of Gibbs free energy as a function of temperature for the reactants, products and each of the four possible transition states. 

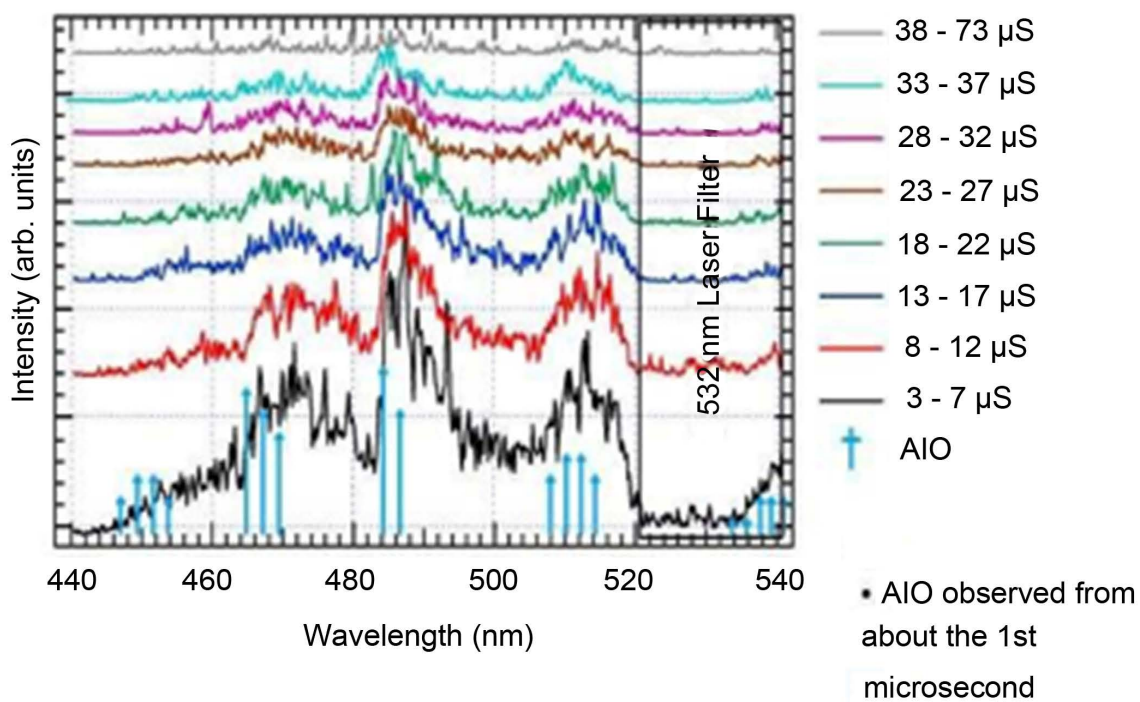

- AlO persists to 41 microseconds

Figure 4. Emission spectrum of the laser-initiated chemical reaction between $\mathrm{Al}$ and $\mathrm{Fe}_{2} \mathrm{O}_{3}$.

$\mathrm{AlO}+2 \mathrm{FeO}+\mathrm{Al}$. The energy of the reactants and the transition state change with the temperature and pressure conditions in the system and thus, the Gibbs free energy is calculated as a function of both the pressure and temperature., as shown in equation.

$$
\begin{aligned}
\Delta G\left(P^{\prime}, T^{\prime}\right)= & \Delta H\left(P=P^{o}, 298 \mathrm{~K}\right)-T^{\prime} \Delta S\left(P=P^{o}, 298 \mathrm{~K}\right) \\
& +\int_{T=298 \mathrm{~K}}^{T^{\prime}} \Delta C_{p} \mathrm{~d} T-T^{\prime} \int_{T=298 \mathrm{~K}}^{T^{\prime}} \frac{\Delta C_{p}}{T} \mathrm{~d} T+\int_{P=P^{o}}^{P^{\prime}}(\Delta V)_{T^{\prime}} \mathrm{d} P
\end{aligned}
$$

\section{Results from an Application}

The identified transition states were used to numerically obtain shock induced chemical reactions, in a system consisting of aluminum and iron oxide, under impact. The numerical solutions were obtained for mixture equations, under one dimensional strain conditions. The impact was from a steel rod. The mixture constitutive equations were used in the framework of extended non-equilibrium thermodynamics. The partial differential equations were numerically integrated using MUSCL scheme. The complete details are published in a separate paper. For completeness of this paper, one result is presented in the following Figure 5.

As can be seen in the figure, the temperature in the thermite mixture of aluminum and iron oxide increases with the impact velocity, due to the shock induced chemical reaction that is modeled using transition states, modified Arrhenius equation and non-equilibrium thermodynamics. When the reaction is completed between aluminum and iron oxide, through the transition state, the final reaction products are iron and aluminum oxide. In the figure, iron is tracked, for increase in impact velocity. 


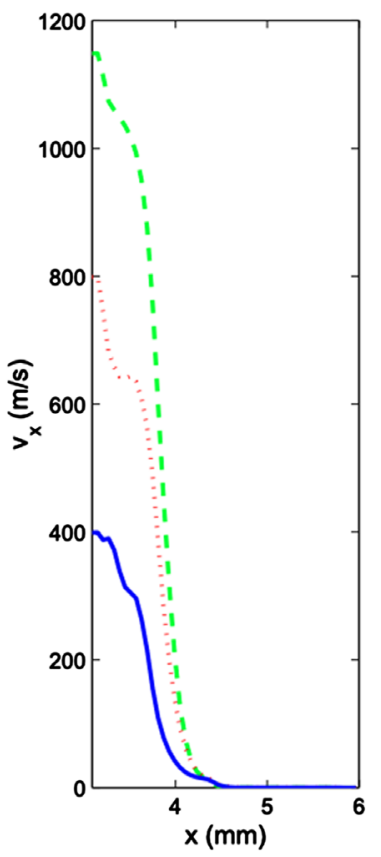

(a)

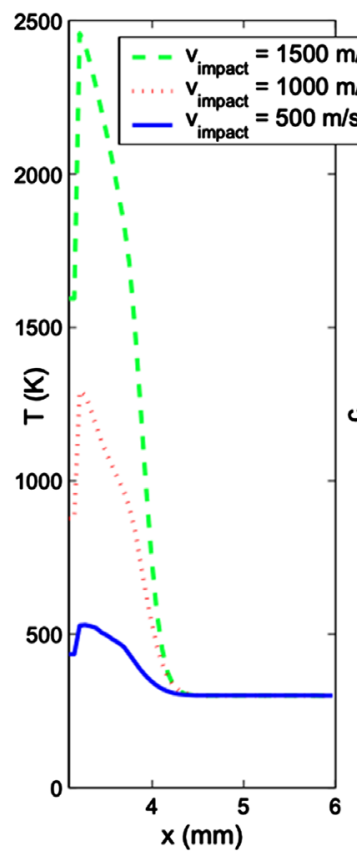

(b)

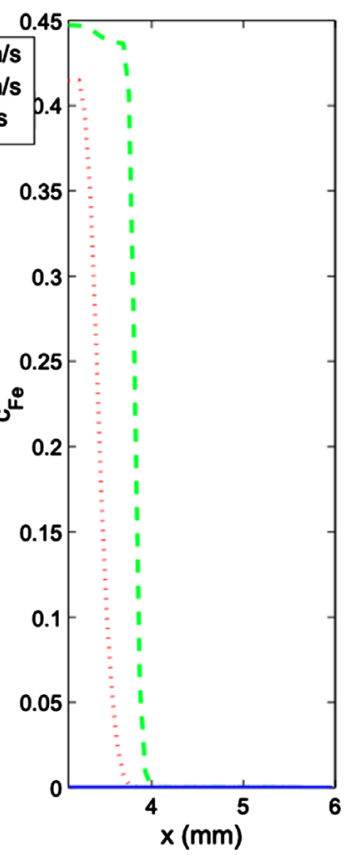

(c)

Figure 5. Impact of a steel projectile on the mixture of aluminum and iron oxide. (a) Velocity; (b) Temperature; (c) Concentration of iron.

\section{Conclusion}

Even though, the mechanisms of shock induced reactions are not clearly understood, transition states are needed to numerically study shock induced chemical reactions in a continuum and mesoscale. In this paper, the transition states are identified for shock induced chemical reactions of mixtures of aluminum and iron oxide. A numerical result for the shock induced chemical reactions, resulting from the impact of a projectile on a mixture of aluminum and iron oxide, using the transition states and non-equilibrium thermodynamics is presented.

\section{Conflicts of Interest}

The authors declare no conflicts of interest regarding the publication of this paper.

\section{References}

[1] Liu, X., et al. (2019) $\mathrm{pH}$ Effects on the Electrochemical Reduction of $\mathrm{CO}_{(2)}$ towards $\mathrm{C}_{2}$ Products on Stepped Copper. Nature Communications, 10, Article No. 32. https://doi.org/10.1038/s41467-018-07970-9

[2] Duan, Z. and Henkelman, G. (2018) Calculations of the pH-Dependent Onset Potential for CO Electrooxidation on Au(111). Langmuir, 34, 15268-15275. https://doi.org/10.1021/acs.langmuir.8b03644

[3] Horie, Y., et al. (1985) Synthesis of Nickel-Aluminide under High Pressure Shock Loading. Materials Letters, 3, 354-359. https://doi.org/10.1016/0167-577X(85)90075-8

[4] Song, T. and Thadhani, N.N. (1993) Synthesis of Nickel = Aluminum Compounds 
by Shock Reaction. Journal of Materials Synthesis and Processing, 1, 347-357.

[5] Thadhani, N.N., et al. (1997) Shock Induced Chemical Reaction of Ti-Si Mixtures of Different Morphologies. Journal of Applied Physics, 82, 1113-1124.

[6] Tomasi, R. and Mann, A.Z. (2007) Effect of Particle Size on the Reaction Propagation in the Composite System of Aluminum Oxide, Zinc Oxide and Nb. Combustion and Flame, 131, 198-207.

[7] Zhu, T., et al. (2002) Reaction Mechanism of Combustion Synthesis of Ni-Al. Materials Science and Engineering, $A$, 329-331, 57-68. https://doi.org/10.1016/S0921-5093(01)01549-0

[8] Horie, Y. and Kipp, M.E. (1988) Modeling of Shock-Induced Chemical Reactions in Powder Mixtures. Journal of Applied Physics, 63, 5718-5727.

https://doi.org/10.1063/1.340309

[9] Bennet, L.S., et al. (1992) Ultrafast Chemical Reactions between Nickel and Aluminum Powders during Shock Loading. Applied Physics Letters, 61, 520-521. https://doi.org/10.1063/1.107874

[10] Yang, Y., et al. (1997) Shock Induced Chemical Reaction in Ni-Al Powder Mixtures. Applied Physics Letters, 70, 3365-3367. https://doi.org/10.1063/1.119172

[11] Zaharieva, R. (2012) Ab Intio Studies of Equations of States and Chemical Reactions in Reactive Structural Materials. PhD Thesis, Georgia Institute of Technology, Atlanta.

[12] Royal, T.E., et al. (1996) Mech and Thermodynamic Process Influence on Shock Chemistry of Ti-Si, Ti-Al, and Ti-B. Metallurgica and Materials Transaction A, 27, 1761-1781.

[13] Wang, L.L., et al. (1993) Thermite Reactions: Their Utilization in the Synthesis of Materials. Journal of Materials Science, 28, 3693-3708. https://doi.org/10.1007/BF00353167

[14] Suryanarayana, P. (2020) Supercomputer to Understand Chemical Reactions. https://www.Phanish.ce.gatech.edu

[15] Bosolough, M.B. (1990) A Thermo-Chemical Model for Shock Induced Reaction (Heat Detonations) in Solids. Journal of Chemical Physics, 92, 1839-1847. https://doi.org/10.1063/1.458066

[16] Bastonov, S.S. (1996) Synthesis and Modification of Materials by Shock Wave, Real Time Measurements and Mechanisms of Reactions. Material Science and Engineering $A, 210,57-63$.

[17] Narayanan, V. (2005) Non-Equilibrium Thermodynamics of Multifunctional Energetic Structural Materials. PhD Thesis, Georgia Institute of Technology, Atlanta.

[18] Thiers, L., et al. (2002) Thermal Explosion of Ti-Al System: Influence of Reaction Medium Microstructure. Combustion and Flame, 131, 198-209. https://doi.org/10.1016/S0010-2180(02)00402-9

[19] Xiao, X. and Thadani, N. (2004) Investigation of Shock-Induced Reaction Behavior of As-Blended and Ball-Milled Ni + Ti Powder Mixtures Using Time-Resolved Stress Measurements. Journal of Applied Physics, 96, 2000-2009. https://doi.org/10.1063/1.1773380

[20] Meyers, M., et al. (1995) Effect of Shock Propagation and Plastic Strain on Chemical Reaction of Nb-Si and Mo-Si Systems. Materials Science and Engineering A, 201, 150-158.

[21] Peiris, S.M. (2016) Laser Induced Reaction of Energetic Thermite Composite, APS Shock Symposium, Invited Lecture on Reactive Materials. 